\title{
Exploring valid reference genes for gene expression studies in Brachypodium distachyon by real-time PCR Shin-Young Hong ${ }^{\dagger 1}$, Pil Joon Seo ${ }^{\dagger 1}$, Moon-Sik Yang ${ }^{2}$, Fengning Xiang ${ }^{3}$ and Chung-Mo Park*1
}

Address: ${ }^{1}$ Department of Chemistry, Seoul National University, Seoul, 151-742, Korea , ${ }^{2}$ Division of Biological Sciences and the Research Institute for Bioactive Materials, Chonbuk National University, Jeonju, 561-756, Korea and ${ }^{3}$ School of Life Sciences, Shandong University, Jinan 250100 , Shandong, PR China

Email: Shin-Young Hong - cutesy77@yahoo.co.kr; Pil Joon Seo - dualnt83@snu.ac.kr; Moon-Sik Yang - mskyang@chonbuk.ac.kr; Fengning Xiang - xfn0990@sdu.edu.cn; Chung-Mo Park* - cmpark@snu.ac.kr

* Corresponding author †Equal contributors

Published: 7 November 2008

BMC Plant Biology 2008, 8:II2 doi:10.1 |86/I47|-2229-8-II2
Received: 29 May 2008

Accepted: 7 November 2008

This article is available from: http://www.biomedcentral.com/I47I-2229/8/II2

(c) 2008 Hong et al; licensee BioMed Central Ltd.

This is an Open Access article distributed under the terms of the Creative Commons Attribution License (http://creativecommons.org/licenses/by/2.0), which permits unrestricted use, distribution, and reproduction in any medium, provided the original work is properly cited.

\begin{abstract}
Background: The wild grass species Brachypodium distachyon (Brachypodium hereafter) is emerging as a new model system for grass crop genomics research and biofuel grass biology. A draft nuclear genome sequence is expected to be publicly available in the near future; an explosion of gene expression studies will undoubtedly follow. Therefore, stable reference genes are necessary to normalize the gene expression data.
\end{abstract}

Results: A systematic exploration of suitable reference genes in Brachypodium is presented here. Nine reference gene candidates were chosen, and their gene sequences were obtained from the Brachypodium expressed sequence tag (EST) databases. Their expression levels were examined by quantitative real-time PCR (qRT-PCR) using 21 different Brachypodium plant samples, including those from different plant tissues and grown under various growth conditions. Effects of plant growth hormones were also visualized in the assays. The expression stability of the candidate genes was evaluated using two analysis software packages, geNorm and NormFinder. In conclusion, the ubiquitin-conjugating enzyme 18 gene $(U B C / 8)$ was validated as a suitable reference gene across all the plant samples examined. While the expression of the polyubiquitin genes (Ubi4 and Ubil 0 ) was most stable in different plant tissues and growth hormone-treated plant samples, the expression of the S-adenosylmethionine decarboxylase gene (SamDC) ranked was most stable in plants grown under various environmental stresses.

Conclusion: This study identified the reference genes that are most suitable for normalizing the gene expression data in Brachypodium. These reference genes will be particularly useful when stress-responsive genes are analyzed in order to produce transgenic plants that exhibit enhanced stress resistance.

\section{Background}

Quantification of gene expression levels is a fundamental step in virtually all aspects of molecular biological research. It is particularly important when genes that are expressed specifically under certain growth conditions are to be compared. Common experimental techniques used 
to evaluate gene expression levels include Northern blot hybridization and reverse transcription (RT)-mediated PCR (RT-PCR); both techniques are practical for analyzing expression of a small set of genes and are complemented by microarray analysis, which is routinely employed for large-scale, global expression profilings. In recent years, the qRT-PCR has been the method of choice for measuring gene expression levels in multiple samples involving a limited number of genes. It provides accurate and sensitive quantification of gene transcript levels, even for those genes with fairly low transcript levels are [1-3].

The popularity of the qRT-PCR method reflects the need for ways to quantitatively analyze gene expression data in the fields of molecular medicine, biotechnology, microbiology, and molecular diagnostics [4] as well as to accurately quantify mRNA levels in plants [5]. However, to avoid experimental deviations or the errors that inevitably occur during sample preparation procedures and data analysis, all of which make quantitation of gene transcripts unreliable, normalization of the qRT-PCR data is essential. The most common way to normalize the data is to use appropriate internal reference genes. An ideal reference gene is expressed at a constant level in all plant tissues under various growth conditions. Its expression should not be influenced by environmental changes or by exogenous application of growth hormones.

Commonly used reference genes in plant molecular biology, frequently called housekeeping genes, play a general role in basic cellular processes, such as cell structure maintenance and primary cellular metabolism, and thus are usually unaffected by external factors. For example, genes encoding tubulins, actins, or elongation factors are widely used in gene expression studies in Arabidopsis thaliana (Arabidopsis hereafter) and Oryza sativa (rice hereafter).

Brachypodium is a temperate wild grass species. Its morphological, genomic, and molecular genetic characteristics and simple growth requirements make it an ideal model system for grass biology [6]. Its genome size (approximately $355 \mathrm{Mbp}$ ) is smaller than that of rice. It also has a short growth cycle. A single plant life cycle can be completed within 6-8 weeks. Self-fertility, the availability of several diploid accessions, and its intrinsic resistance to diverse biotic and abiotic stress conditions are additional attributes.

Arabidopsis is widely used as a model system for all flowering plants. Rice has been used as a model for genomics research for all temperate grass species, including major cereals, such as barley and wheat. However, recent genomic analyses have revealed that the Brachypodium genome is phylogenetically closer to the genomes of economically important crops, such as wheat and barley, and several potential biofuel grasses, including switch grasses [7]. Brachypodium exhibits many agricultural traits similar to these grass crops. Furthermore, a protocol has recently been established for infecting Brachypodium with Magnaporthe grisea (rice blast) in order to explore its potential as a model system in which to study host-pathogen interactions [8]. To date, such molecular assays have routinely been carried out using rice or related monocots.

The rapidly growing interest in Brachypodium triggered the establishment of a series of genomic resources; these include the collecting of nuclear genomic sequences and bacterial artificial chromosome (BAC)/EST-based libraries in addition to the characterization of individual genetic resources [9]. Furthermore, the Brachypodium genome project is currently underway, and the full genomic sequence will be released to the public in the near future [7]. Several groups have also reported the existence of efficient Agrobacterium-mediated Brachypodium transformation systems $[10,11]$, which are essential for functional genomics studies in Brachypodium. Such technological progress in Brachypodium research can help realize its potential as a new model system for biofuel grass biology, provided that stable reference genes are identified for gene expression studies in this plant species.

In this study, we report on reference genes that can potentially be used to normalize the results of gene expression studies in Brachypodium. Nine candidate genes, including those encoding actin (ACT7), elongation factor 1alpha (EF1 $\alpha$, glyceraldehyde 3-phosphate dehydrogenase (GAPDH), rubisco activase (RCA), SamDC, alpha-tubulin (TUA6), UBC18, Ubi4, and Ubi10, were chosen, and partial gene sequences were extracted from the available Brachypodium databases http://harvest.ucr.edu. Brachypodium was either grown under various stress conditions or treated with an array of growth hormones. Different plant tissues and plants at different developmental stages were also included in the assays. Our data showed that while expression of the UBC18 gene was the most stable among all the plant samples examined, the Ubi4 and Ubi10 genes would be appropriate for analysis of gene expression studies in different plant tissues. Their expressions were also stable under the growth hormone treatments. In the meantime, the SamDC gene showed the most stable expression patterns in plants grown under diverse abiotic stress conditions. This work will certainly facilitate our future work on gene expression studies in biofuel grass crops as well as in Brachypodium.

\section{Results}

Selection of potential reference genes in Brachypodium

A handful of reference genes, including those encoding tubulins, actins, and elongation factors, are regularly used to normalize the RT-PCR or qRT-PCR data in Arabidopsis 
and rice. Additional genes, such as elongation initiation factor $4 a$ (ELF4a), are sometimes employed to further standardize the data on plant samples grown under specific growth conditions. However, because none of the reference genes currently used in Arabidopsis and rice provides a stable expression under all the examined conditions, multiple reference genes must be included to ensure the normalization of the data in some molecular analyses.

Although genomic, cellular, and morphological aspects of Brachypodium have been widely explored in recent years, little information is available about molecular genetic analyses, and thus no systematic efforts have been made to identify its stable reference genes. Extensive literature searches have revealed that genes encoding various types of proteins, including enzymes and structural proteins, are widely used as internal controls for studies of plant gene expression through RT-PCR, qRT-PCR, and gene chip assays $[12,13]$. Analyzing these reference genes in other plant species led us to choose nine candidate reference genes in Brachypodium; ACT7, RCA, EF1 $\alpha$, GAPDH, SamDC, TUA6, UBC18, Ubi4, and Ubi10. Using the sequence information deposited in the HarvEST http:// harvest.ucr.edu under the GenBank accession numbers listed in Table 1, a series of qRT-PCR primers was designed which the sizes of the PCR products were approximately $100-300$ bp (see Additional file 1).

\section{RNA integrity and amplification specificity}

For qRT-PCR analyses, we included 21 different total RNA samples extracted from various plant materials. The plant materials analyzed included 5 different plant tissues (callus, leaf, root, spike, stem), plants at 4 different developmental stages (early vegetative, late vegetative, transition, and reproductive phases), and plants exposed to 4 different abiotic stress conditions, such as high salinity (300 $\mathrm{mM} \mathrm{NaCl})$, cold $\left(4^{\circ} \mathrm{C}\right.$ for $\left.5 \mathrm{~h}\right)$, heat $\left(42^{\circ} \mathrm{C}\right.$ for $\left.2 \mathrm{~h}\right)$, and drought (400 $\mathrm{mM}$ mannitol). Brachypodium seedlings were also treated with 8 different growth hormones; indole-3-acetic acid (IAA, $50 \mu \mathrm{M}$ ), brassinolide (BL, 50 $\mu \mathrm{M})$, zeatin $(50 \mu \mathrm{M})$, abscisic acid (ABA, $100 \mu \mathrm{M})$, gib- berellic acid (GA, $50 \mu \mathrm{M})$, 1-aminocyclopropane-1-carboxylic acid (ACC, $50 \mu \mathrm{M}$ ), salicylic acid (SA, $100 \mu \mathrm{M}$ ), and methyl jasmonic acid (MeJA, $100 \mu \mathrm{M}$ ).

Prior to carrying out qRT-PCR reactions, the viability of all RNA samples was examined using RT-PCR to evaluate the expression of the GAPDH gene. All the RT-PCR reactions produced a single GAPDH-specific band with a predicted molecular size (approximately $500 \mathrm{bp}$ ) on a 1.2\% agarose gel visualized with ethidium bromide staining (see Additional file 2), confirming the hypothesis that the RNA samples extracted from Brachypodium plant materials are appropriate for transcript level analysis. The integrity and quality of RNA samples were further evaluated by electrophoretic analysis using the Labwork Image Acquisition and Analysis software (Media Cybernetics) (see Additional file 2).

The results showed that the GAPDH gene was stably expressed under various abiotic stress conditions and not discernibly affected by diverse growth hormones, although it exhibited less stability in some plant tissues (see Additional file 2).

To examine the stability of reference gene expressions, transcript levels were measured by qRT-PCR. Aliquots of the primary cDNA synthesis reaction mixture were used in qRT-PCR to amplify gene-specific primer pairs of each of the nine candidate reference genes. Primer dimer formation and nonspecific amplification can falsely increase gene transcript levels and thus must be avoided, especially when qRT-PCR is carried out using the SYBR green dyes. Gene-specific amplification of each of the nine candidate genes was confirmed by the appearance of a single, dominant peak in the qRT-PCR dissociation curve analyses (see Additional files 3, 4, 5, 6, 7, 8, 9, 10, 11).

\section{Identification of marker genes for growth hormone treatments}

A set of marker genes that are regulated specifically by growth hormones and environmental factors is a prereq-

Table I: Selected reference genes used for gene expression studies in Brachypodium

\begin{tabular}{lllll}
\hline Gene symbol & Gene name & Brachypodium & Rice & Arabidopsis \\
\hline ACT7 & Actin 7 & DV478555 & LOC_OsI0g36650 & AT5G098I0 \\
EFI $\alpha$ & Elongation factor I-alpha & DV482887 & LOC_Os03g08050 & AT5G60390 \\
GAPDH & Glyceraldehyde-3-phosphate dehydrogenase & DV482924 & LOC_Os08g03290 & AT3G04I20 \\
RCA & Rubisco activase & DV482669 & LOC_OsIIg47970 & AT2G39730 \\
SamDC & S-adenosyl methionine decarboxylase & DV482676 & LOC_Os04g42090 & AT3G25570 \\
TUA6 & Tubulin alpha-6 & DV478602 & LOC_OsIIgI4220 & AT4GI4960 \\
UBCI8 & Ubiquitin-conjugating enzyme 18 & DV48I689 & LOC_OsI2g44000 & AT5G42990 \\
Ubi4 & Polyubiquitin (Ubi4) & DV482834 & LOC_Os04g53620 & AT5G20620 \\
Ubil0 & Polyubiquitin (Ubil0) & DV484269 & LOC_Os06g46770 & AT4G05320 \\
\hline
\end{tabular}

The Brachypodium gene accession numbers refer to the dbEST division of GenBank [25]. The Arabidopsis Genome Initiative (AGI) codes and the TIGR rice genome locus identifiers of the gene homologues are also listed. 
uisite for determining reference gene expression stability under various experimental conditions. Since there is no information on marker genes available in Brachypodium, we screened potential marker genes in the Brachypodium databases that are homologous to those well characterized in Arabidopsis.

Transcript levels of the selected marker genes were compared by qRT-PCR in Brachypodium plants treated either with various growth hormones or with abiotic stresses (see Additional file 12). We found that Brachypodium genes, which are homologous to IAA1, ARR4, BAS1, NPR1, CBF3, and HSC70, were significantly induced by the relevant treatments. In contrast, genes that are homologous to GA3OX2-1, RD22, and Chitinase1 were repressed under the conditions examined (see Additional files 13 and 14). These observations indicate that treating Brachypodium seedlings with growth hormones or growing seedlings under abiotic stress conditions conferred regulatory effects on the selected marker genes similar to those found in Arabidopsis.

\section{Expression data analysis}

A simple, widely used way to identify stably expressed genes is to calculate cycle threshold (Ct) values in the qRTPCR reactions. The $\mathrm{Ct}$ value represents the cycle at which a significant increase of the PCR product, which is measured by an increase in fluorescence, occurs; this cycle is generally marked by the middle of the exponential phase of amplification. Gene expression analyses of the nine reference genes exhibited a narrow mean $\mathrm{Ct}$ value range across all the experimental samples (Figure 1 ). The $\mathrm{Ct}$ values ranged from 13 to 22, while most of the values were distributed between 15 and 19. Ubi4 was the most abundantly transcribed, reaching the threshold fluorescence peak after 13 amplification cycles. The Ct average of all other genes was approximately 17 cycles. As a result, the Ubi4 transcript level was approximately 16 times more abundant than the average of the data set. The least abundant transcripts were those of $U B C 18$ having a Ct value of 19 or higher. Most of the reference genes had relatively smaller variations in gene expression (below 1 cycle) except for $R C A$.

The geNorm software was employed as a means of determining the expression stability of the selected reference genes http://medgen.ugent.be/ jjvdesomp/genorm/[14]. This program automatically calculates the average expression stability value $(\mathrm{M})$ as the average pairwise variation (V) of a particular gene with all other control genes and determines the $\mathrm{V}$ values with all other control genes as the standard deviation of the logarithmically transformed expression ratios.

Figure 2 displays the $M$ values of remaining reference genes at each step during stepwise exclusion of the least

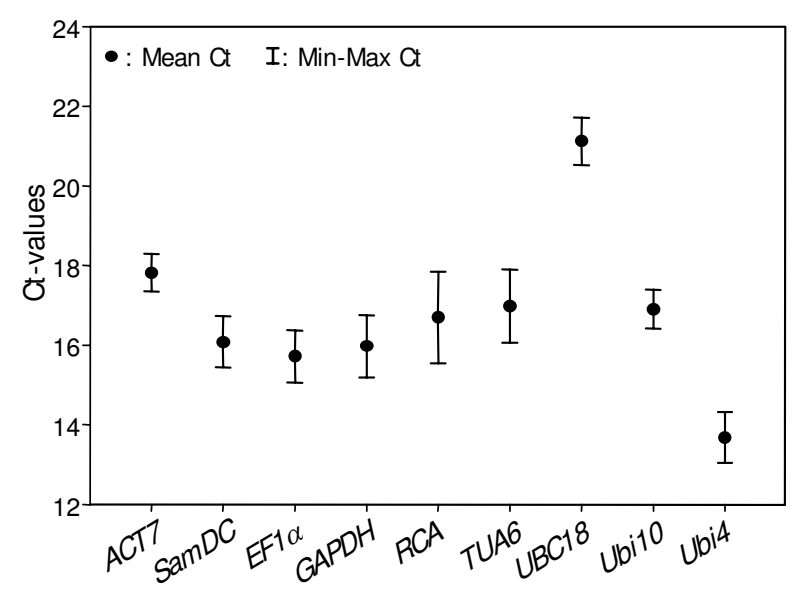

Figure I

Expression levels of candidate reference genes in different plant samples. The scatter plots exhibit the expression levels of candidate reference genes in the tested Brachypodium samples $(n=21)$. Values are given as cycle threshold numbers (Ct values) with a mean of duplicate samples. Bars indicate standard error of the mean.

stable reference gene. Starting from the least stable gene on the left, the genes are ranked according to increasing expression stability, ending with the two most stable genes on the right. The most stable reference gene was not identical in individual plant samples. While the SamDC and $U B C 18$ genes were most stably expressed throughout the plant life cycle (Figure 2b), the Ubi4 and Ubi10 genes ranked highest in different plant tissue samples with an $\mathrm{M}$ value of 0.169 (Figure 2c). For the growth hormonetreated samples, the most stable genes were GAPDH and $U B C 18$ with an $\mathrm{M}$ value of 0.169 (Figure 2d). The $E F 1 \alpha$, $U B C 18$, and SamDC genes ranked high in Brachypodium seedlings grown under abiotic stress conditions, indicating that these genes are stably expressed and probably play a housekeeping role (Figures $2 \mathrm{e}$ and $2 \mathrm{f}$ ). All the tested samples reached a high expression stability with relatively low $M$ values of less than 1 , which are far below the default limit of $M \leq 1.5$ [14].

Examining all the expression data examined revealed that UBC18 and ACT7 are the most stable genes that might be widely used for multiple purposes (Figure 2a). In contrast, $R C A$ was the least stable among the genes examined.

To determine the optimal number of reference genes required for accurate normalization, the geNorm software was used to calculate the pairwise variation $\left(\mathrm{V}_{n} / \mathrm{V}_{\mathrm{n}+1}\right)$ between the sequential normalization factors (NF) $\left(\mathrm{NF}_{\mathrm{n}}\right.$ and $\mathrm{NF}_{\mathrm{n}+1}$ ) (Figure 3 ). In the original publication describing geNorm [14], a threshold of 0.15 or lower for the pairwise variation was proposed to indicate that inclusion of 
(a) Total

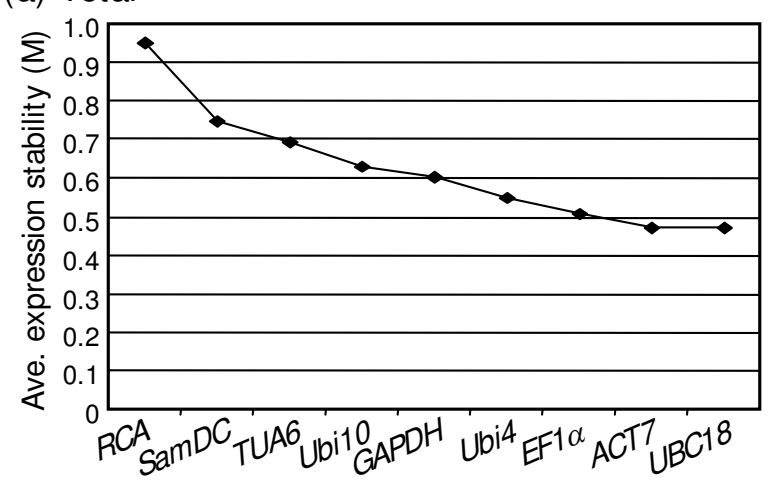

(c) Tissue

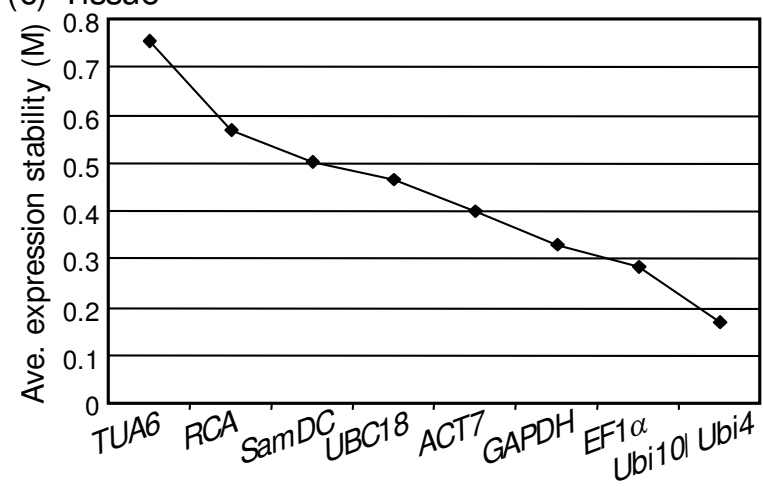

(e) Heat/Cold

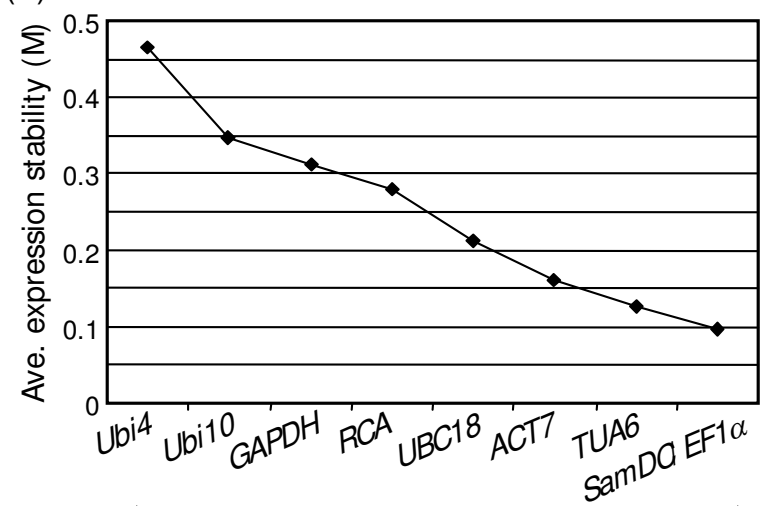

4.... Least stable genes Most stable genes: (b) Developmental stage

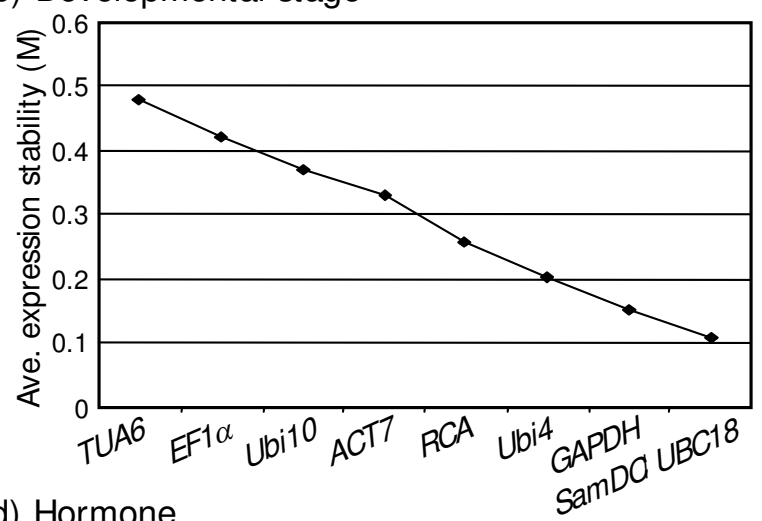

(d) Hormone

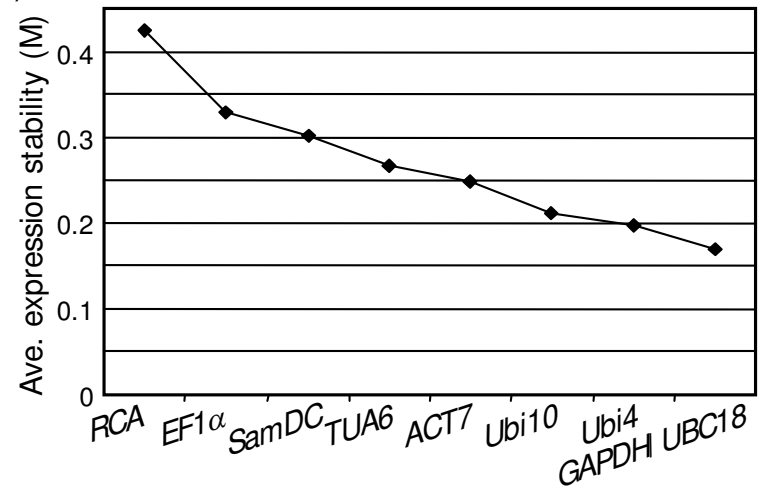

(f) Salt/Drought

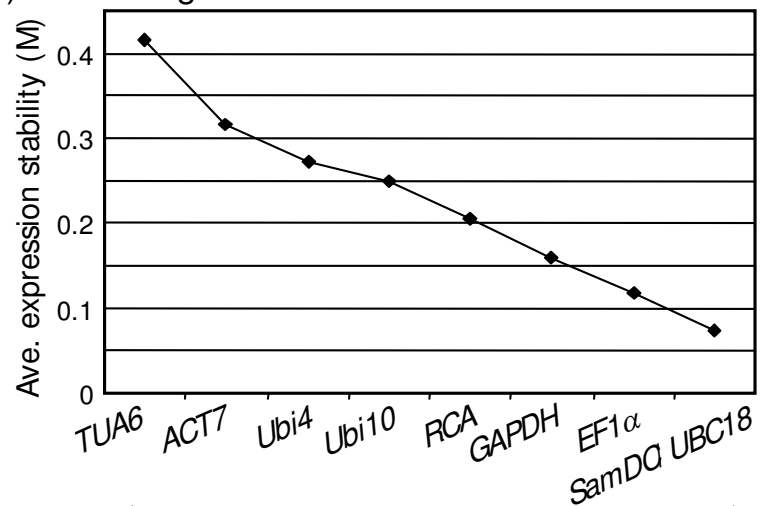

.... Least stable genes Most stable genes.

\section{Figure 2}

Average expression stability values $(M)$ of the candidate reference genes. Average expression stability values (M) of the reference genes were measured during stepwise exclusion of the least stable reference genes. A lower $M$ value indicates more stable expression, as analyzed by the geNorm software in plant samples at different developmental stages (b), plant tissue samples (c), hormone-treated samples (d), heat- and cold-treated samples (e), and in high salt- and drought-treated samples (f). The $M$ values calculated for all plant samples examined are also given (a). 
an additional reference gene is unnecessary. However, 0.15 is not a strict cut-off value but an ideal value. Therefore, the graph displayed in Figure 3 is intended only to provide guidance for determining the optimal number of reference genes. In addition, the observed trend of changing $\mathrm{V}$ values when using additional genes can be equally informative. Therefore, in most cases, the use of three reference genes results in much more accurate and reliable normalization than only one gene and is thus considered to be a valid normalization strategy. We therefore decided to take the threshold of 0.1 as a cut-off value for the inclusion of our reference genes.

Analysis of the data obtained from different plant tissues using the geNorm software produced a plot displaying the average expression stability values of the candidate reference genes in each round of the analysis, ranking them from the least stable gene at the far left to the two most stable genes at the far right (Figure 2). Evaluation of different plant tissues revealed a significant decrease in the pairwise variation with the inclusion of a fourth gene (note the difference in the $\mathrm{V}$ values of $\mathrm{V} 2 / 3$ and $\mathrm{V} 3 / 4$ in Figure 3 ). The pairwise variation at the $\mathrm{V} 3 / 4$ value was 0.086 , which is below the threshold of 0.1 , indicating that the normalization factor should preferably contain at least three reference genes. Therefore, it would be ideal to include Ubi4, Ubi10, and EF1 $\alpha$ as reference genes to normalize gene expression data in plant tissues.

Analysis of the pairwise variation in the growth hormonetreated samples revealed that a V score of 0.064 is achieved with two reference genes (Figures $2 \mathrm{~d}$ and 3 ), indicating that the average of the top two reference genes, GAPDH and $U B C 18$, is the optimal normalization factor for gene expression studies in the growth hormonetreated Brachypodium plant materials. In the heat- and cold-treated plant samples, SamDC and EF1 $\alpha$ were the most stable reference genes (Figure 2e). As in the growth hormone-treated samples, the pairwise variation at V2/3 was below the threshold of 0.1 , and thus two reference genes, SamDC and EF1 $\alpha$, would be sufficient (Figure 3).

In the meantime, evaluation of the salt- and droughttreated samples did not exhibit any discernible difference in the pairwise variation with the inclusion of a third gene (Figures $2 \mathrm{f}$ and 3 ). It is apparent that the average of the top two reference genes, SamDC and UBC18, is the optimal normalization factor for gene expression studies in the salt- and drought-treated samples.

To further confirm the results obtained by the geNorm program, we also employed the Normfinder software http://www.mdl.dk/publicationsnormfinder.htm, an algorithm for identifying the optimal normalization gene among a set of candidate genes. This software ranks the set of candidate normalization genes according to the stability of their expression patterns in a given sample set analyzed in a given experimental design [15]. The results of the Normfinder analysis applied to our data sets are summarized in Table 2. The ranking of the reference genes was almost identical to that determined by the geNorm software. The Ubi4 and Ubi10 genes still ranked highest for different plant tissues and growth hormone-treated plant samples, while the GAPDH or SamDC genes appeared to

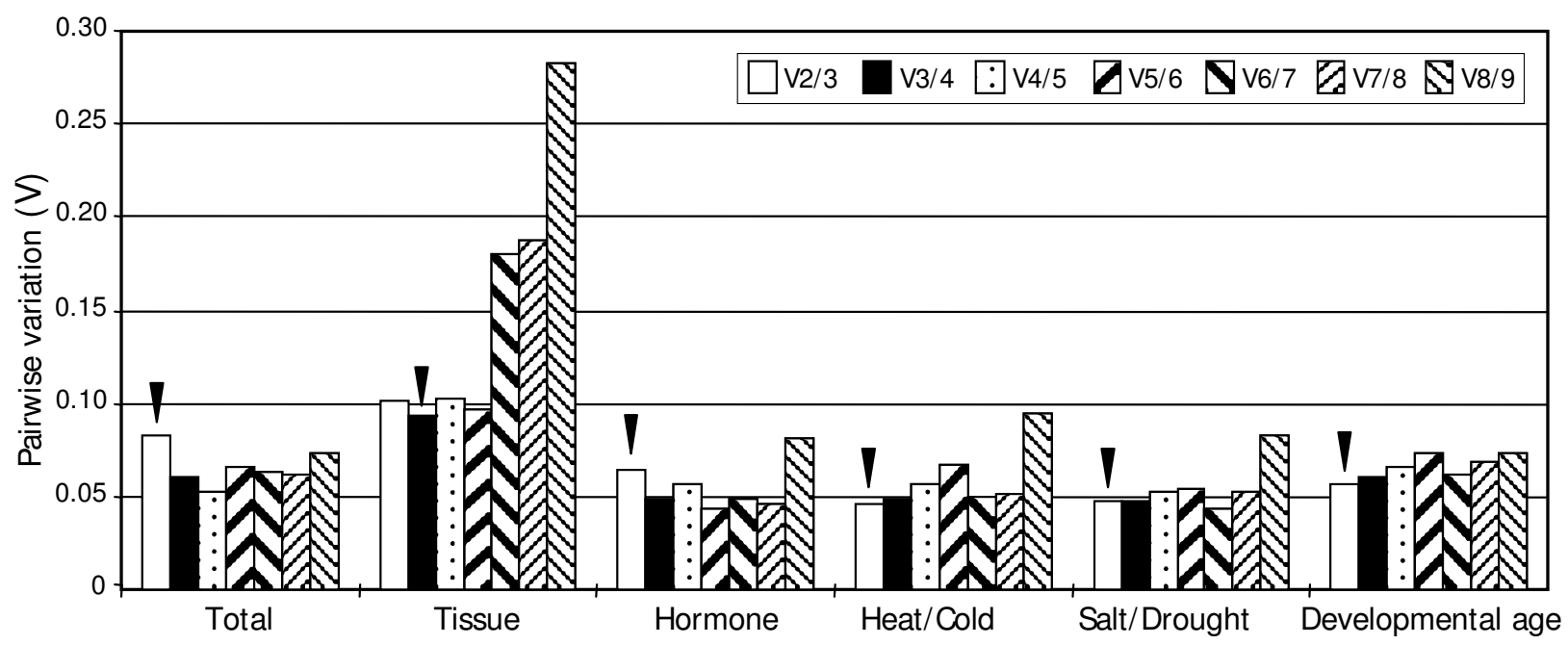

Figure 3

Pairwise variation $(V)$ analysis of the candidate reference genes. The pairwise variation $\left(V_{n} / V_{n+1}\right)$ was analyzed between the normalization factors $\mathrm{NF}_{n}$ and $\mathrm{NF}_{n+1}$ by the geNorm software to determine the optimal number of reference genes required for qRT-PCR data normalization. 
be the highest reliable controls for plant samples exposed to abiotic stress.

\section{Discussion}

The qRT-PCR is broadly accepted as the method of choice for accurate and sensitive quantification of gene transcript levels, even for those genes whose transcript levels are low. For valid qRT-PCR analysis, accurate normalization of gene expression against an appropriate internal control is required. Most gene expression studies in the literature generally use a single internal control for normalization [16], and the validity of the conclusion depends highly on the control applied. Therefore, it is necessary to validate the expression stability of the control gene under specific experimental conditions prior to its use for normalization.

The most commonly used reference genes in the pregenomic era were chosen primarily for their known or suspected housekeeping roles in basic cellular processes. The actin gene, one such reference gene, is used in normalizing the quantification of gene expression levels [17]. Genes encoding GAPDH, actin, and EF1 $\alpha$ have been used as the most relevant reference genes for berry development [18]. However, the actin gene was found to be inappropriate for use as a reference gene, since some variations of the transcript levels were observed in different plant tissues and plants grown under different growth conditions.

Our study showed that the polyubiquitin genes (Ubi4 and Ubi10) and the UBC18 gene encoding an ubiquitin-conjugating enzyme exhibited the most stable expression in different plant tissues or in plants treated with various growth hormones. These results are also supported by the previous observation in which Ubi10 showed a highly stable expression pattern in Arabidopsis [12]. An ubiquitin gene $(U B Q)$ and a TUA gene are the most stably expressed in plant tissue samples from poplar [12]. Notably, the $U B Q$ gene is the most stably expressed in the parasitic plant Orobanche ramose [19]. On the other hand, the expression of $U B Q 5$ and $E F 1 \alpha$ was the most stable among all the plant tissues of rice [20]. In potato, $E F 1 \alpha$ is also the most stably expressed in plants grown under biotic and abiotic stress conditions [21]. In addition, GAPDH exhibited the most stable expression in different plant tissues of sugarcane [22]. Although genes encoding ubiquitins and polyubiquitins are stably expressed in different plant tissues in several plant species, these genes may not be stably expressed in other plant species. The reference genes appear to be regulated differentially in different plant species and cell types or under different experimental conditions. Therefore, no single reference gene with stable expression is suitable for normalizing all expression data obtained from various experimental conditions.

Our data demonstrate that $U B C 18$ and SamDC are a reliable set of reference genes for normalizing gene expression data when multiple Brachypodium samples are analyzed (Figure 2). In particular, UBC18 showed acceptable expression stability in all the plant samples examined. Accumulation of the SamDC transcripts is ubiquitous in different plant organs in Brachypodium, including the callus, spike, root, leaf, and sheath. Therefore, it is not surprising that SamDC seems to be essential for embryo development in Arabidopsis [23]. It is likely that GAPDH and SamDC play a housekeeping role in Brachypodium growth and development.

While the geNorm software is used to identify not only the most stable gene but also the optimum pair of genes with least variation in their expression ratios, the NormFinder software is able to identify the gene with the most stable expression. Our results lead us to propose that the mean data rendered by UBC18, SamDC, Ubi4, and Ubi10 be used to normalize gene expression values in Brachypodium more accurately. To our knowledge, this is the first systematic attempt to identify reference genes in Brachypodium.

Table 2: Brachypodium reference genes for normalization and their expression stability values calculated by the NormFinder software

\begin{tabular}{|c|c|c|c|c|c|c|c|c|c|c|c|c|}
\hline \multirow[t]{2}{*}{ Rank } & \multicolumn{2}{|c|}{ Tissue } & \multicolumn{2}{|c|}{ Growth hormone } & \multicolumn{2}{|c|}{ Heat/Cold } & \multicolumn{2}{|c|}{ High salt/Drought } & \multicolumn{2}{|c|}{ Developmental stage } & \multicolumn{2}{|c|}{ Total } \\
\hline & Gene & Stability & Gene & Stability & Gene & Stability & Gene & Stability & Gene & Stability & Gene & Stability \\
\hline 1 & Ubi4 & 0.0846 & Ubilo & 0.1377 & GAPDH & 0.1723 & SamDC & 0.0372 & Ubi4 & 0.0987 & UBCI8 & 0.2586 \\
\hline 2 & Ubilo & 0.0846 & Ubi4 & 0.1640 & EFI $\alpha$ & 0.1951 & UBCI8 & 0.0378 & SamDC & 0.0996 & Ubi4 & 0.3363 \\
\hline 3 & GAPDH & 0.2751 & GAPDH & 0.1768 & SamDC & 0.2164 & GAPDH & 0.0738 & UBCI8 & 0.156 & $E F I \alpha$ & 0.3876 \\
\hline 4 & $E F I \alpha$ & 0.2796 & ACT7 & 0.2053 & ACT7 & 0.2434 & $E F I \alpha$ & 0.1951 & GAPDH & 0.2276 & SamDC & 0.4791 \\
\hline 5 & ACT7 & 0.3708 & TUA6 & 0.2103 & $R C A$ & 0.2689 & $R C A$ & 0.2063 & ACT7 & 0.3401 & ACT7 & 0.4845 \\
\hline 6 & $U B C / 8$ & 0.5032 & UBCI 8 & 0.2291 & Ubilo & 0.3139 & Ubilo & 0.3426 & $R C A$ & 0.3682 & TUAG & 0.5777 \\
\hline 7 & SamDC & 0.5512 & $E F I \alpha$ & 0.3028 & TUA6 & 0.3279 & ACT7 & $0.344 I$ & Ubilo & 0.3776 & Ubilo & 0.5937 \\
\hline 8 & $R C A$ & 0.8238 & SamDC & 0.3946 & UBCI8 & 0.3309 & Ubi4 & 0.4078 & $E F / \alpha$ & 0.6029 & GAPDH & 0.6658 \\
\hline 9 & TUAG & 1.3712 & $R C A$ & 0.7124 & Ubi4 & 0.8483 & TUAG & 0.7401 & TUAG & 0.6579 & $R C A$ & 0.926 \\
\hline
\end{tabular}

In each column, a low stability value as an estimate of the combined intra- and intergroup variation of the respective gene represents a high gene expression stability of the respective gene between the matched samples. 
The Brachypodium genome project is currently underway under the auspices of the US Department of Energy. A draft genomic sequence is expected to be released in the near future, which will trigger diverse molecular genetic studies on Brachypodium as well as on related grass species. Of particular interest is how the plant responds to environmental stresses. However, there is no report so far on the stress-responsive genes or those involved in growth hormone signaling in Brachypodium. In this work, we searched for potential marker genes mediating stress responses and growth hormone signaling. Most of the selected marker genes exhibited responses to abiotic stresses and growth hormones that were similar to those of the Arabidopsis gene homologues, suggesting that the known stress-responsive genes and underlying molecular mechanisms in Arabidopsis are conserved in Brachypodium. One exception is the Brachypodium RD22 gene. While the Arabidopsis RD22 gene (AtRD22) is induced by $\mathrm{ABA}$ and abiotic stresses, the Brachypodium $R D 22$ gene is repressed by ABA, high salt, and drought (see Additional files 13 and 14). At least some Brachypodium genes, such as $R D 22$, likely play a role that is characterized by molecular mechanisms distinct from those exerted by the Arabidopsis gene homologues.

\section{Conclusion}

We identified several reference genes that are suitable for qRT-PCR data normalization in Brachypodium. This is the first systematic exploration of valid reference genes in this plant species. Although most of the selected candidate genes exhibited stable expression patterns acceptable for reference genes, different genes showed the highest stability in different plant samples. While expression of Ubi4 and Ubi10 was most stable in different plant tissues and growth hormone-treated plant samples, that of SamDC was most stable under different environmental conditions. Overall, $U B C 18$ is our reference gene of choice for analyzing multiple Brachypodium samples.

\section{Methods}

\section{Plant sample preparation}

A community standard diploid inbred line of Brachypodium distachyon, $\mathrm{Bd} 21$, was used for all experimental treatments. The palea and lemma were carefully peeled off from the mature seeds with fine forceps. The stripped seeds were sterilized by soaking in a solution of $10 \%$ household bleach $(5.25 \% \mathrm{NaOCl})$ supplemented with $0.1 \%$ Tween 20 for 10 min with occasional rocking. The sterilized seeds were thoroughly rinsed three times with sterile double-distilled water and placed on Murashige and Skoog (MS)-agar plates (MS-agar plates hereafter) ( $4.3 \mathrm{~g} / \mathrm{l} \mathrm{MS}$ salts with vitamins, 3\% sucrose, $\mathrm{pH} 5.8$, and $0.4 \%$ phytagel). The seeds were cold-treated at $4{ }^{\circ} \mathrm{C}$ for 2 days to synchronize germination and allowed to germinate in a culture room controlled at $25^{\circ} \mathrm{C}$ with a daily photoperiodic cycle of $16 \mathrm{~h}$ light and $8 \mathrm{~h}$ dark.
Four-week-old Brachypodium plants were used for treatments with growth hormones and abiotic stresses. Harvested plant samples were frozen in liquid nitrogen and stored at $-80^{\circ} \mathrm{C}$ until RNA extraction. For gene expression studies in different plant tissues, appropriate plant tissues were harvested from 5-week-old, fully-grown plants. For gene expression studies at different developmental stages, plants were harvested at 7 days after germination (DAG) (early vegetative phase), 12 (late vegetative phase), 20 (transition phase), and 30 (reproductive phase) (see Additional file 2).

\section{Growth hormones and abiotic stresses}

For growth hormone treatments, 4-week-old Brachypodium plants were transferred to the MS liquid cultures supplemented with IAA $(50 \mu \mathrm{M})$, brassinolide (BL, 50 $\mu \mathrm{M})$, zeatin $(50 \mu \mathrm{M})$, ABA $(100 \mu \mathrm{M})$, GA $(50 \mu \mathrm{M}), 1$-aminocyclopropane-1-carboxylic acid (ACC, $50 \mu \mathrm{M}$ ), SA (100 $\mu \mathrm{M})$, or with MeJA $(100 \mu \mathrm{M})$ and incubated for $5 \mathrm{~h}$ with gentle shaking. The mock seedlings were similarly incubated in a MS liquid but without adding growth hormones.

For salt and drought stress treatments, 4-week-old Brachypodium plants were transferred to the MS liquid cultures supplemented either with $300 \mathrm{mM} \mathrm{NaCl}$ or with $400 \mathrm{mM}$ mannitol, respectively, and gently shaken for $5 \mathrm{~h}$. For cold and heat treatments, the seedlings were incubated at $4{ }^{\circ} \mathrm{C}$ for $5 \mathrm{~h}$ or $42^{\circ} \mathrm{C}$ for $2 \mathrm{~h}$, respectively.

\section{Selection of potential reference genes in Brachypodium}

To identify potential Brachypodium homologues of the Arabidopsis or rice genes commonly used as internal controls for gene expression studies, we queried Version 0.52 of the HarvEST:Brachypodium software http://harvestweb.org, which displays 6 different libraries from Brachypodium. All gene sequences were obtained from the GenBank dbEST database. The HarvEST:Brachypodium software contains best BLASTX hits from the UniProt and the rice and Arabidopsis genomes (TIGR version 5, February 2007, and TAIR version 7, April 2007, respectively).

The HarvEST is principally an EST database-viewing software that emphasizes gene function and is oriented to comparative genomics and design of oligonucleotides, with an aim to support diverse research activities, such as microarray content design, functional annotation, and physical and genetic mapping http://harvest.ucr.edu. The HarvEST:Brachypodium is the most recent and standardized EST database for Brachypodium and thus can be used to examine sequence alignments and determine where individual sequences reliably deviate from a consensus sequence.

Selected Brachypodium ESTs of potential reference genes were used to design primers. A set of qRT-PCR primers 
with high-efficiency for nine individual reference genes was designed using the Primer3 software (version 0.4.0) [24]. The primers were designed to have melting temperatures in a range of $50-60^{\circ} \mathrm{C}$, depending on individual genes. Their sequences are summarized in Additional file 1.

\section{Total RNA extraction and primary CDNA synthesis}

Total RNA was extracted from appropriate plant samples using the RNeasy Plant mini kit (Qiagen, Valencia, CA) according to the manufacturer's procedure. The quality and integrity of the RNA samples were evaluated by absorbance measurements and by electrophoretic analysis using the Labwork Image Acquisition and Analysis Program (Media Cybernetics, San Diego, CA). All the RNA samples used in the qRT-PCR reactions showed a 260/280 $\mathrm{nm}$ absorbance ratio of $1.8-2.2$. RNA samples with a ratio of $\approx 2$ are generally qualified for subsequent enzymatic reactions. Prior to RT-PCR and qRT-PCR, total RNA samples were pretreated with a RNase-free DNase I to eliminate any contaminating genomic DNA. The primary cDNA was synthesized from approximately $3 \mu \mathrm{g}$ of total RNA using the MMLV first-strand synthesis system (Promega, Madison, WI) and the oilgo-dT and random primers in a reaction volume of $40 \mu \mathrm{l}$ according to the manufacturer's procedure.

To rule out any genomic DNA contamination in the RNA preparations, the RNA samples and genomic DNA were subject in parallel to PCR amplifications of the ARR4 and GAPDH gene sequences ( 30 cycles), and the PCR products were compared. No visible amplifications of genomic DNAs were detected from the RNA samples (see Additional file 15).

\section{RT-PCR and qRT-PCR}

One $\mu$ l of the primary cDNA synthesis reaction mixture was taken for subsequent PCR amplification by RT-PCR or qRT-PCR. RT-PCR runs were routinely carried out for 20 to 35 cycles, depending on the linear range of PCR amplification for each gene. Each PCR cycle included incubations at $94^{\circ} \mathrm{C}$ for $30 \mathrm{~s}$, at $55^{\circ} \mathrm{C}$ for $1 \mathrm{~min}$, and at $72^{\circ} \mathrm{C}$ for $5 \mathrm{~min}$. One additional cycle at $72^{\circ} \mathrm{C}$ for $10 \mathrm{~min}$ was run after the last cycle to allow trimming of incomplete polymerizations. Positive and negative control genes were included in the reaction sets to ensure the feasibility of the assay conditions. The RT-PCR primers used are listed in Additional file 1.

qRT-PCR reactions were carried out in 96-well blocks with an Applied Biosystems 7500 Real-Time PCR System using the SYBR Green I master mix in a reaction volume of 25 $\mu \mathrm{l}$, which contains $1 \mu \mathrm{l}$ of the primary cDNA reaction mixture, 2X SYBR Green PCR Master Mix (Applied Biosystems, Foster City, CA), and a primer pair. The primers used are listed in Additional file 1. The two-step thermal cycling profile used was $15 \mathrm{~s}$ at $95^{\circ} \mathrm{C}$ and $1 \mathrm{~min}$ at $60^{\circ} \mathrm{C}$. All qRT-PCR reactions were carried out in biological duplicates, each of which was used for RNA extraction followed by qRT-PCR in triplicate. The final threshold cycle $\left(C_{t}\right)$ values were the mean of six values (biological duplicates, each with triplicate). The comparative $\Delta \Delta \mathrm{C}_{\mathrm{t}}$ method was used to evaluate the relative quantities of each amplified product in the samples. The $\mathrm{C}_{\mathrm{t}}$ was automatically determined for each reaction by the Applied Biosystems 7500 Real-Time PCR System set with default parameters. The specificity of the qRT-PCR reactions was determined by melt curve analysis of the amplified products using the standard method installed in the System.

Each qRT-PCR reaction set included a negative control with water instead of cDNA. Duplicate measurements were averaged, and the mean values were used for further calculations.

\section{Determining expression stability of reference genes}

The expression stability of each reference gene was analyzed using the geNorm (version 3.5) and NormFinder (version 0.953) software packages, which are also integrated into the GenEx software (version 4.3.5, http:// www.multid.se). The geNorm software calculates the gene expression stability $(\mathrm{M})$ for a reference gene as the average pairwise variation $\mathrm{V}$ for the gene with all other tested reference genes. Stepwise exclusion of the gene with the highest $M$ value allows the tested genes to be ranked according to the stability of their expression patterns.

The NormFinder software is an algorithm for identifying the optimal normalization gene among a set of candidate genes. It ranks the set of candidate normalization genes according to the stability of their expression patterns in a given sample set under a given experimental design. Therefore, it can analyze expression data obtained through any quantitative method, such as qRT-PCR and microarray-based expression profiling. The lowest stability value represents the most stable gene expression within the gene set examined.

\section{Abbreviations}

ACT7: actin 7; Ct value: cycle threshold; EF1 $\alpha$ : elongation factor 1-alpha; EST: expressed sequence tag; GAPDH: glyceraldehyde-3-phosphate dehydrogenase; qRT-PCR: quantitative real-time RT-PCR; RT-PCR: reverse transcriptionPCR; RCA: rubisco activase; SamDC: S-adenosylmethionine decarboxylase proenzyme; TUA6: tubulin alpha-6; UBC18: ubiquitin-conjugating enzyme 18; Ubi10: Polyubiquitin (Ubi10); Ubi4: Polyubiquitin (Ubi4).

\section{Authors' contributions}

SYH and PJS performed all the experimental procedures and statistical calculations jointly. FX established the culture conditions for Brachypodium and provided technical 
support. MSY provided technical assistance and scientific discussion. CMP conceived the project, supervised the study design, and contributed to writing the manuscript.

\section{Additional material}

\section{Additional file 1}

PCR primers used in this work. The primers were designed using the Primer3 software (version 0.4.0) [24]. They had melting temperatures in a range of $50-60^{\circ} \mathrm{C}$, depending on individual genes. ${ }^{*}$ The primer set was used only for RT-PCR analysis of GAPDH. All other primers except for this were used for QRT-PCR reactions.

Click here for file

[http://www.biomedcentral.com/content/supplementary/14712229-8-112-S1.doc]

\section{Additional file 2}

RT-PCR analyses of GAPDH transcript levels in different Brachypodium samples. Total RNA samples were isolated from plants grown under abiotic stresses (a), from different plant tissues (b), from plants treated with various growth hormones $(c)$, or from plants at different developmental stages (d). The quality of the RNA samples was determined by electrophoretic analysis using the Labwork Image Acquisition and Analysis Program (Media Cybernetics, see Methods).

Click here for file

[http://www.biomedcentral.com/content/supplementary/14712229-8-112-S2.ppt]

\section{Additional file 3}

Dissociation curve data for UBC18 in growth hormone-treated samples.

Click here for file

[http://www.biomedcentral.com/content/supplementary/1471 2229-8-112-S3.ppt]

\section{Additional file 4}

Dissociation curve data for Ubi4 in growth hormone-treated samples. Click here for file

[http://www.biomedcentral.com/content/supplementary/14712229-8-112-S4.ppt]

\section{Additional file 5}

Dissociation curve data for Ubi10 in growth hormone-treated samples.

Click here for file

[http://www.biomedcentral.com/content/supplementary/1471-

2229-8-112-S5.ppt]

\section{Additional file 6}

Dissociation curve data for RCA in growth hormone-treated samples. Click here for file

[http://www.biomedcentral.com/content/supplementary/14712229-8-112-S6.ppt]

\section{Additional file 7}

Dissociation curve data for TUA6 in growth hormone-treated samples.

Click here for file

[http://www.biomedcentral.com/content/supplementary/14712229-8-112-S7.ppt]

\section{Additional file 8}

Dissociation curve data for GAPDH in growth hormone-treated samples.

Click here for file

[http://www.biomedcentral.com/content/supplementary/14712229-8-112-S8.ppt]

\section{Additional file 9}

Dissociation curve data for $\mathrm{EF} 1 \alpha$ in growth hormone-treated samples. Click here for file

[http://www.biomedcentral.com/content/supplementary/14712229-8-112-S9.ppt]

\section{Additional file 10}

Dissociation curve data for SamDC in growth hormone-treated samples.

Click here for file

[http://www.biomedcentral.com/content/supplementary/14712229-8-112-S10.ppt]

\section{Additional file 11}

Dissociation curve data for ACT7 in growth hormone-treated samples. Click here for file [http://www.biomedcentral.com/content/supplementary/14712229-8-112-S11.ppt]

\section{Additional file 12}

Brachypodium marker genes for gene expression studies in response to growth hormone treatments and abiotic stresses. The Brachypodium genes homologous to the Arabidopsis or rice genes that are regulated by growth hormones or abiotic stresses were examined by qRT-PCR. The sources of the gene accession numbers are those described in Table 1. Click here for file [http://www.biomedcentral.com/content/supplementary/14712229-8-112-S12.doc]

\section{Additional file 13}

qRT-PCR data on Brachypodium genes regulated by growth hormones and abiotic stresses. Transcript levels were measured by qRT-PCR. Bars mark the standard error of the mean. The mean values were used as positive controls to determine the effects of growth hormone and abiotic stresses on reference genes. The gene accessions and the effects of each treatment on individual genes were summarized in Additional file 12. Click here for file [http://www.biomedcentral.com/content/supplementary/14712229-8-112-S13.ppt]

\section{Additional file 14}

Validation of the most suitable gene UBC18 using plants treated with $A B A$ or high salt. RNA samples isolated from plants treated with $A B A$ or high salt, as used in Additional file 13, were subject to RT-PCR of the UBC1 8 and TUA genes. The RT-PCR products were evaluated by electrophoretic analysis using the Labwork Image Acquisition and Analysis Program (Media Cybernetics).

Click here for file

[http://www.biomedcentral.com/content/supplementary/14712229-8-112-S14.ppt] 


\section{Additional file 15}

Genomic PCR and RT-PCR amplifications of the ARR4 and GAPDH genes. (a) The ARR4 and GAPDH gene structures. The gene sequences were extracted from the JGI 4 X Brachy Sequence produced by the US Department of Energy Joint Genome Institute http://www.jgi.doe.gov/. The gene structures were predicted using the GENSCAN server http:// genes. mit.edu/GENSCAN.html. The black boxes denote exons. The numbers in parentheses indicate the sizes of exons and introns in base pairs (bp). The arrowheads mark the position and direction of the PCR primers. (b) Genomic PCR and RT-PCR. The PCR reactions were carried out for 30 cycles using either genomic DNA (gDNA) or primary cDNA (cDNA) synthesized from total RNA pretreated with a RNase-free DNase I. The PCR primers were those listed in Additional file 1. The numbers in parentheses indicate the sizes of PCR products in $b p$. The arrow marks a predicted PCR product. SM, size marker.

Click here for file

[http://www.biomedcentral.com/content/supplementary/14712229-8-112-S15.ppt]

\section{Acknowledgements}

This work was supported by Brain Korea 2I, Biogreen 21 (2008040 1034006), and National Research Laboratory Programs and by grants from the Plant Signaling Network Research Center, Korea Research Foundation (2005-070-C00 29), and from Korea Science and Engineering Foundation (2007-03415). We thank Emily Wheeler for editorial assistance.

\section{References}

I. Bustin SA: Real-time, fluorescence-based quantitative PCR: a snapshot of current procedures and preferences. Expert Rev Mol Diagn 2005, 5:493-498.

2. Bustin SA, Benes V, Nolan T, Pfaffl MW: Quantitative real-time RT-PCR - a perspective. J Mol Endocrinol 2005, 34:597-60I.

3. Nolan T, Hands RE, Bustin SA: Quantification of mRNA using real-time RT-PCR. Nat Protoc 2006, I:1559-1582.

4. Bustin SA, Dorudi S: Molecular assessment of tumour stage and disease recurrence using PCR-based assays. Mol Med Today 1998, 4:389-396.

5. Gachon C, Mingam A, Charrier B: Real-time PCR: what relevance to plant studies? J Exp Bot 2004, 55: | 1445- |454.

6. Garvin DF, Gu YQ, Hasterok R, Hazen SP, Jenkins G, Mockler TC, Mur LJ, Vogel JP: Development of Genetic and Genomic Research Resources for Brachypodium distachyon, a New Model System for Grass Crop Research. Crop Sci 2008, 48:S69-S84.

7. Opanowicz M, Vain P, Draper J, Parker D, Doonan JH: Brachypodium distachyon: making hay with a wild grass. Trends Plant $\mathrm{Sci}$ 2008, 13:172-177.

8. Parker D, Beckmann M, Enot DP, Overy DP, Rios ZC, Gilbert M, Talbot N, Draper J: Rice blast infection of Brachypodium distachyon as a model system to study dynamic host/pathogen interactions. Nat Protoc 2008, 3:435-445.

9. Huo N, Lazo GR, Vogel JP, You FM, Ma Y, Hayden DM, ColemanDerr D, Hill TA, Dvorak J, Anderson OD, Luo MC, Gu YQ: The nuclear genome of Brachypodium distachyon: analysis of BAC end sequences. Funct Integr Genomics 2008, 8: I35-147.

10. Pãcurar DI, Thordal-Christensen $\mathrm{H}$, Nielsen KK, Lenk I: A highthroughput Agrobacterium-mediated transformation system for the grass model species Brachypodium distachyon $\mathbf{L}$. Transgenic Res 2007. DOI 10.1007/s I I248-007-9|59-y.

11. Vain P, Worland B, Thole V, McKenzie N, Alves SC, Opanowicz M, Fish LJ, Bevan MW, Snape JW: Agrobacterium-mediated transformation of the temperate grass Brachypodium distachyon (genotype Bd2I) for T-DNA insertional mutagenesis. Plant Biotechnol J 2008, 6:236-245.
12. Czechowski T, Stitt M, Altmann T, Udvardi MK, Scheible WR: Genome-wide identification and testing of superior reference genes for transcript normalization in Arabidopsis. Plant Physiol 2005, 139:5-17.

13. Brunner AM, Yakovlev IA, Strauss SH: Validating internal controls for quantitative plant gene expression studies. BMC Plant Biol 2004, 4: | 4-20.

14. Vandesompele J, De Preter K, Pattyn F, Poppe B, Van Roy N, De Paepe A, Speleman F: Accurate normalization of real-time quantitative RT-PCR data by geometric averaging of multiple internal control genes. Genome Biol 2002, 3:34.I-34.II.

15. Andersen CL, Jensen JL, Orntoft TF: Normalization of real-time quantitative reverse transcription-PCR data: a model-based variance estimation approach to identify genes suited for normalization, applied to bladder and colon cancer data sets. Cancer Res 2004, 64:5245-5250.

16. Suzuki T, Higgins PJ, Crawford DR: Control selection for RNA quantitation. Biotechniques 2000, 29:332-337.

17. Staiger C): Signaling to the actin cytoskeleton in plants. Annu Rev Plant Physiol Plant Mol Biol 2000, 5 I:257-288.

18. Reid KE, Olsson N, Schlosser J, Peng F, Lund ST: An optimized grapevine RNA isolation procedure and statistical determination of reference genes for real-time RT-PCR during berry development. BMC Plant Biol 2006, 6:27-37.

19. González-Verdejo CI, Die JV, Nadal S, Jiménez-Marín A, Moreno MT, Román B: Selection of housekeeping genes for normalization by real-time RT-PCR: analysis of Or-MYBI gene expression in Orobanche ramosa development. Anal Biochem 2008, 379:176- I8I.

20. Jain M, Nijhawan A, Tyagi AK, Khurana JP: Validation of housekeeping genes as internal control for studying gene expression in rice by quantitative real-time PCR. Biochem Biophys Res Commun 2006, 345:646-65I.

21. Nicot N, Hausman JF, Hoffmann L, Evers D: Housekeeping gene selection for real-time RT-PCR normalization in potato during biotic and abiotic stress. J Exp Bot 2005, 56:2907-2914.

22. Iskandar HM, Simpson RS, Casu RE, Bonnett GD, Maclean DJ, Manners JM: Comparison of reference genes for quantitative realtime polymerase chain reaction analysis of gene expression in sugarcane. Plant Mol Biol Rep 2004, 22:325-337.

23. Ge C, Cui X, Wang Y, Hu Y, Fu Z, Zhang D, Cheng Z, Li J: BUD2, encoding an $S$-adenosylmethionine decarboxylase, is required for Arabidopsis growth and development. Cell Res 2006, 16:446-456.

24. Rozen S, Skaletsky HJ: Primer3 on the WWW for general users and for biologist programmers. In Bioinformatics Methods and Protocols: Methods in Molecular Biology Edited by: Krawetz S, Misener S. Totowa, Humana Press; 2000:365-386.

25. Vogel JP, Gu YQ, Twigg P, Lazo GR, Laudencia-Chingcuanco D, Hayden DM, Donze T], Vivian LA, Stamova B, Coleman-Derr D: EST sequencing and phylogenetic analysis of the model grass Brachypodium distachyon. Theor Appl Genet 2006, I I 3:186-195.

Publish with Bio Med Central and every scientist can read your work free of charge

"BioMed Central will be the most significant development for disseminating the results of biomedical research in our lifetime. "

Sir Paul Nurse, Cancer Research UK

Your research papers will be:

- available free of charge to the entire biomedical community

- peer reviewed and published immediately upon acceptance

- cited in PubMed and archived on PubMed Central

- yours - you keep the copyright

BioMedcentral 